\title{
Needle-free Epicutaneous For t 2 DNA Vaccine is Effective for Preventing and Treating Biting Midge ( Forcipomyia taiwana ) allergy in a murine model
}

\author{
Yi-Hsing Chen ${ }^{1}$, Mey-Fann Lee ${ }^{2}$, Chi-Sheng $\mathrm{Wu}^{1}$, and Shyh-Jye $\mathrm{Lin}^{3}$ \\ ${ }^{1}$ Taichung Veterans General Hospital \\ ${ }^{2}$ Taichung Veterans General Hospital Department of Medical Research \\ ${ }^{3}$ Chung Shan Medical University
}

September 24, 2021

\section{Needle-free Epicutaneous For t 2 DNA Vaccine is Effective for Preventing and Treating Biting Midge (Forcipomyia taiwana) allergy in a murine model}

To the Editor,

Allergen-specific immunotherapy (ASIT) remains the only treatment capable of inducing immune tolerance to the corresponding allergen and potentially treating the root cause of the allergic disease. ${ }^{1}$ As the treatment course of protein-based vaccines for ASIT is time-consuming, an easily administered epicutaneous anti-allergic DNA-based vaccine is an attractive method, especially in light of the COVID-19 pandemic. ${ }^{2}$

The biting midge, Forcipomyia taiwana, is the most prevalent cause of biting insect allergy in Taiwan. It is a tiny hematophagous midge that attacks en masse. As many as $60 \%$ of exposed individuals develop allergic reactions to the bites. ${ }^{3}$ The midge is widely distributed throughout Taiwan and southern China. For t 2 is the most predominant, with $75 \%$ of midge-allergic patients showing specific IgE to For t $2 .{ }^{4}$ Allergic reactions to midge bites are not limited to humans but also seen in livestock, such as horses, cattle, sheep, and donkeys, causing significant veterinarian problems.

E.coli -expressed For t 2 recombinant protein (rFor t 2) was used as an allergen to sensitize and challenge the mice. ${ }^{5}$ For t 2 -encoding fragment (GenBank accession EU678971) was amplified by PCR. The PCR products were subcloned into pVAX1 (Life Technologies, Carlsbad, CA). The experiments were designed using two approaches: therapeutic and prophylactic (Fig 1). The therapeutic approach is to imitate ASIT in human with established allergy while the prophylactic approach to non-allergics. Twenty-five $\mu \mathrm{g}$ For t 2 DNA was determined as the optimal dose after dose-finding experiments (Supplementary Fig S1). For each treatment, the hair of the abdominal area of the mice was removed using a depilatory,tape-stripped,then patched with $25 \mu \mathrm{g}$ For t 2 DNA vaccine for one hour and removed.A total of three treatments were given spaced one week apart (Fig 1 and Fig S2). For t 2 proteins were detected in the patched skin and the immune organ spleen at 24 hoursand had significantly increased at 48 hours after last treatment (Fig S3). Scratch bouts after rFor t 2 challenge were used as a clinical surrogate of itch. We measured For t 2-specific IgE, IgG1 and IgG2a in the sera as well as mRNA and proteins of IL-13, interferon-gamma, IL-10, and FOXP3 in the culture supernatants of splenocytes after stimulation with various doses of $\mathrm{rFor}$ t 2 at 37 for 3-5 days by ELISA and real-time quantitative PCR. Histopathology of the challenged skins was examined.

After epicutaneous DNA vaccination, the allergen-induced itchin both groups significantly improved, and For t 2-specific IgE and IgG1/IgG2a ratio decreased significantly at week 6 or week 8 (Fig 2). Levels of mRNA and protein of IL-13 decreased significantly, but IFN-gamma and IL-10 remained unchanged. Expression of 
FOXP3 mRNA increased (Fig 2, protein data not shown). Eosinophils infiltration in the challenged skin significantly decreased (Fig S4).

This is the first study to demonstrate an epicutaneous needless anti-allergic DNA vaccine that effectively treats an established allergic condition and prevents the development of an allergic disease using biting midge allergy as a model. After epicutaneous DNA vaccination, in addition to allergen-induced itch, the changes of multiple biomarkers suggest that immune tolerance was induced after the epicutaneous DNA vaccine.

Our data show that though the molecular weight of the For t 2 DNA vaccine is as high as 4000 base pairs, it can penetrate the dermal barrier and translate the corresponding protein in the targeted skin and the spleen of the vaccinated mice. It is possible that the DNA vaccine passes the epidermis via the hair follicles as the skin is tape-stripped before epicutaneous vaccination. ${ }^{6}$

The mode of this anti-allergic epicutaneous DNA vaccine may potentially be used in other specific immunotherapies for other allergens.

Mey- Fann Lee ${ }^{1}$

Chi-Sheng $\mathrm{Wu}^{2}$

Shyh-Jye $\operatorname{Lin}^{3}$

Yi-Hsing Chen ${ }^{2,4^{*}}$

${ }^{1}$ Department of Medical Research, Taichung Veterans General Hospital, Taichung, Taiwan

${ }^{2}$ Division of Allergy, Immunology and Rheumatology, Taichung Veterans General Hospital, Taichung, Taiwan

${ }^{3}$ School of Medical Laboratory and Biotechnology, Chung Shan Medical University, Taichung, Taiwan

${ }^{4}$ School of Medicine, National Yang Ming Chiao Tung University, Taipei, Taiwan

\section{Correspondence}

Yi-Hsing Chen, Division of Allergy, Immunology and Rheumatology, Taichung Veterans General Hospital, 1650 Taiwan Boulevard, Section 4, Taichung 40705, Taiwan

Emailysanne@vghtc.gov.tw

\section{Funding information}

Taiwan Ministry of Science and Technology, Grant/Award Number:108-2321-B-010-015 and 109-2327-B-010004

\section{CONFLICTS OF INTEREST}

The authors have no conflicts of interest with regard to this study.

\section{Author contributions}

LMF andCYH designed studies, interpreted the results and wrote the article. WCS performed experiments and analyzed data. LSJ gave technical support and revised the manuscript.

\section{References}

1. Gradman J, Halken S. Preventive Effect of Allergen Immunotherapy on Asthma and New Sensitizations. J Allergy Clin Immunol Pract. 2021.

2. Pfaar O, Agache I, Bonini M,et al. COVID-19 pandemic and allergen immunotherapy - an EAACI survey. Allergy. 2021.

3. Chen YH, Lee MF, Lan JL, et al. Hypersensitivity to Forcipomyia taiwana (biting midge): clinical analysis and identification of major For t 1, For t 2 and For t 3 allergens. Allergy. 2005;60(12):1518-1523. 
4. Chen YH, Hwang GY, Chen PC, et al. Molecular cloning and immunologic characterization of for t 2: a major allergen from the biting midgeForcipomyia taiwana. Allergy. 2011;66(5):703-705.

5. Lee MF, Yang KJ, Wang NM, et al. The development of a murine model for Forcipomyia taiwana (biting midge) allergy. PLoS One. 2014;9(3):e91871.

6. Lademann J, Richter H, Schanzer S, et al. Follicular penetration of nanocarriers is an important penetration pathway for topically applied drugs. Hautarzt. 2019;70(3):185-192.

\section{Figure legends}

Figure 1Schedules of therapeutic (A), prophylactic (B), vaccination and grouping (C) for For t 2 DNA with skin patches. Time points of sensitization with rFor t 2 /alum, videotaping, and sacrificing are indicated.

Figure 2 Scratching bouts, changes in serum For t 2-specific IgE and IgG1/IgG2a ratio, and mRNA expression levels of IL-13, interferon $\gamma(\mathrm{INF}-\gamma)$, IL-10 and Foxp3 in For t 2-stimulated splenocytes from (A) therapeutic and (B) prophylactic groups by real-time PCR. The statistical significance of differences between groups was assessed by the Bonferroni multiple range test. ${ }^{*}$ denotes $\mathrm{p}<0.05$, ${ }^{* *}$ denotes $\mathrm{p}<0.01$, ns denotes not statistically significant.

Figure S1(A) Dose finding schedule of For t 2 DNA patch vaccine on rFor t 2-sensitized mice in the pilot study. (B) Counts of scratching bouts from each group of mice before (day 0) and after (day 61) challenged by intradermal injection of rFor t 2. (C) Total IgE and For t 2-specific IgG2a antibodies in the sera at week 9 determined by ELISA. * denotes $p<0.05$, **denotes $p<0.01$, *** denotes $p<0.001$, ns denoted not statistically significant by the Bonferroni multiple range test.

Figure S2 The representative skin images show before (A) and after (B) patch application. The For t 2 DNA vaccine was delivered onto the pre-shaved abdominal skin through a Fin chamber for 1 hour.

Figure S3 H\&E and immunohistochemotry (IHC) staining using rabbit anti-For t 2 polyclonal antibody of skin and spleen sections under 400x light microscope. For t 2 protein expression of skin and spleen were examed 24 and 48 hours after the last treatment of epicutaneous vaccination.

Figure S4 Effects of For t 2 vaccine on histopathology of challenged skins from (A) therapeutic or (B) prophylactic groups by H\&E staining. Figures showed the representative abdominal skin sections obtained 48 hours after intradermal challenge under $100 \mathrm{x}$ and $400 \mathrm{x}$ light microscope. Moreover, the infiltrating inflammatory cells were quantified under 400 -fold view from each group. The statistical significance of differences between groups was assessed by the Bonferroni multiple range test. ${ }^{* *}$ denoted $\mathrm{p}<0.01$, ns denotes not statistically significant.

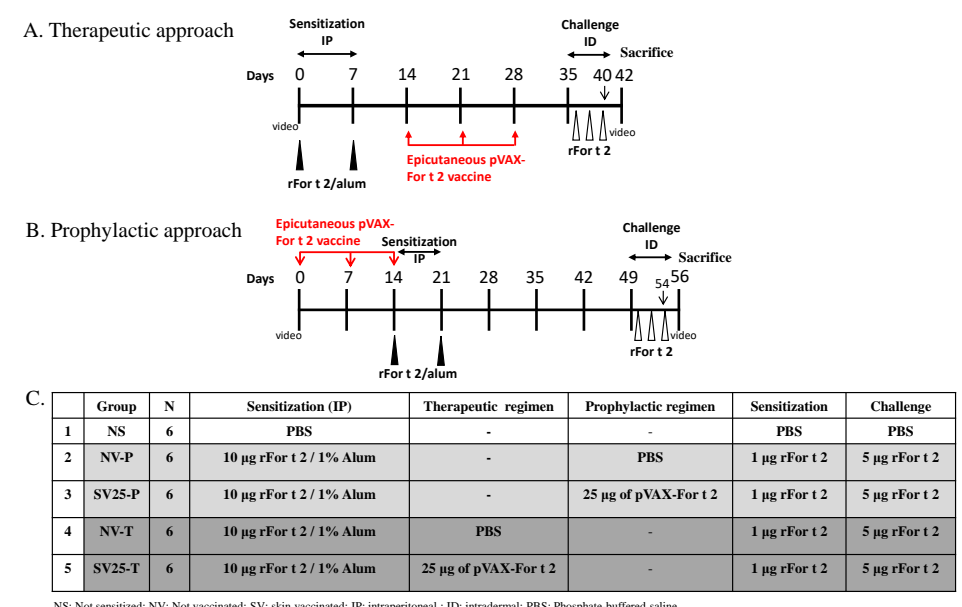



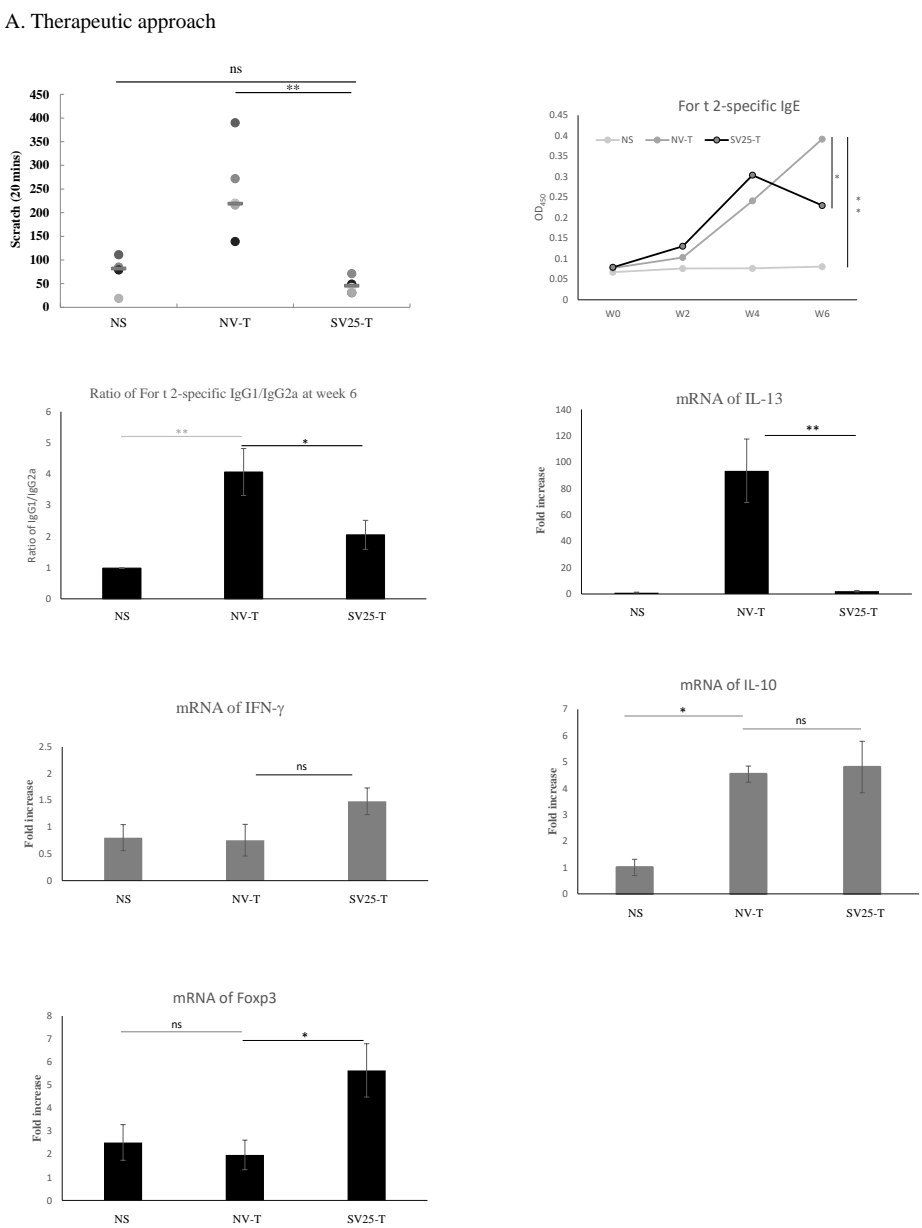
B. Prophylactic approach
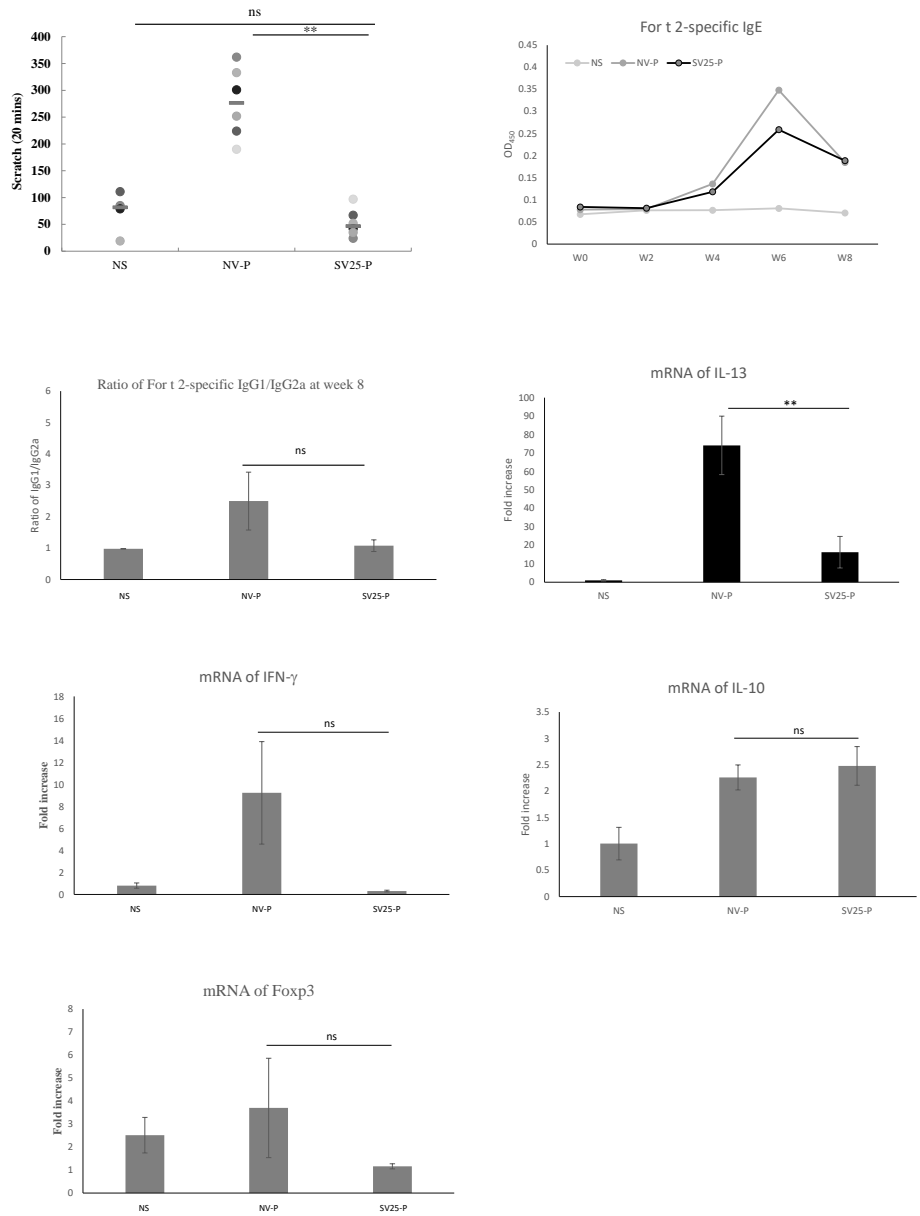